\title{
Screening Colonoscopy: Still the Best Choice, but for How Long?
}

\author{
Yoji Takeuchi
}

Published online: 24 September 2013

(c) Springer Science+Business Media New York 2013

Colonoscopy is one of the most reliable methods of colorectal cancer (CRC) screening since it not only detects asymptomatic early CRC, but also precancerous lesions such as colorectal adenomas. Based on the accepted adenoma-carcinoma sequence, removal of colorectal adenomas decreases CRC incidence and mortality [1, 2]. Although no randomized, controlled trials support the hypothesis that total colonoscopy decreases CRC incidence and mortality, several cohort studies reported that colonoscopy plus polypectomy decreased CRC incidence. The National Polyp Study reported that colonoscopy decreased the development of CRC and mortality from CRC, estimating that the removal of all detected colorectal polyps would prevent $76-90 \%$ of CRCs and $53 \%$ of CRCassociated deaths $[1,2]$. Based on these and other findings, clinical guidelines have recommended colonoscopy as a screening modality for CRC [3, 4]. Moreover, screening colonoscopy reduced CRC-associated mortality at a relatively low incremental cost [5]. Thus, colonoscopic screening can provide great benefit for patients at risk of CRC.

Despite these benefits, colonoscopy has several drawbacks. First, colonoscopy is more expensive and timeconsuming than fecal immunochemical testing with or without added sigmoidoscopy. Second, even though subjects undergoing colonoscopy must ingest large volumes of polyethylene glycol or a similar laxative to prepare the mucosa for visualization, bowel preparation is inadequate in up to $25 \%$ of patients at the time of their examination

\section{Y. Takeuchi $(\square)$}

Department of Gastrointestinal Oncology, Osaka Medical Center for Cancer and Cardiovascular Diseases, 1-3-3 Nakamichi, Higashinari-ku, Osaka 537-8511, Japan

e-mail: takeuti-yo@mc.pref.osaka.jp
[6]. Poor bowel preparation can result in an incomplete examination, including inability to reach the cecum and overlooking some lesions. Third, the discomfort and pain that can be experienced during colonoscopy is one of the main causes of low adherence to the procedure. Fourth, about one-third of patients experience procedure-related discomfort after colonoscopy [7], which affects their willingness to undergo additional colonoscopy. Finally, some patients experience severe adverse events during and after the colonoscopy, including cardiopulmonary deterioration, bowel perforation, hemorrhage, infection, and post polypectomy syndrome. Thus, although beneficial, colonoscopy with or without polypectomy is accompanied by adverse effects. Since screening procedures by definition involve asymptomatic patients, the risk threshold is low. Moreover, the negative predictive value of a neoplasm-free mucosa in an adequately prepared subject is such that subsequent colonoscopic screening is essentially unnecessary [8].

In the study published by Czwornog et al. [9] in this issue of Digestive Diseases and Sciences, the authors concluded that normal-weight females (particularly those under age 60) have the lowest adenoma prevalence rate (APR) but have longer procedure times and require higher amounts of sedation. Those results, consistent with previous reports and our clinical practice, indicated that the APR in such subjects was $17.9 \%$, meaning that colonoscopic screening is beneficial for only the $17.9 \%$ of normal weight females younger than 60 years old, but can be harmful for the remaining $82.1 \%$ due to longer procedural times and the greater use of sedatives. According to their findings, the risk/benefit is excessive for screening such a population. Colonoscopic screening was only recommended for populations with high APRs such as males, obese individuals, and subjects aged $\geq 60$ years. Also, the 
authors recommend that less-invasive screening methods should be considered for subjects at low CRC risk, proposing a tailored approach using fecal occult blood testing or sigmoidoscopy. In Japan, we utilize fecal immunochemical test (FIT) for primary CRC screening and risk stratification for the entire screening population, due to the cost benefit and lack of a sufficient number of colonoscopists, although the superiority of FIT to TCS is not datadriven. Colonoscopy is then recommended for FIT positive subjects with a positive predictive value for CRC detection, including intramucosal carcinoma, of $\sim 5 \%$, although the adherence rate is low. This imperfect approach might be enhanced by the adoption of more advanced detection methods such as fecal exfoliated DNA tests, computed tomographic colonography, and positron emission tomography.

In the study published by Czwornog et al. [9], the authors evaluated the diagnostic efficacy weighed against the unsuitableness of colonoscopy using gender, age, and weight as differentiators. Despite their conclusion that colonoscopy was less efficacious and more risky [9] for younger women with normal BMI than for their male counterparts, colonoscopy may remain the optimal screening modality compared with other methods. For example, a comparison of hypothetical flexible sigmoidoscopy with total colonoscopy reported that the former could detect lesions in only $35 \%$ of women with proximal neoplasia [10], suggesting that colonoscopy may be the optimal CRC screening tool for women. Thus, data should support recommendations of alternate screening modalities for the less at-risk group (i.e., younger women with normal BMI in this study). In Japan, for example, the most common cause of cancer death in females is CRC; accordingly, deviation from current screening guidelines is not recommended.

In summary, although Czwornog and Austin reported subject populations at higher risk/benefit for colonoscopic CRC screening, their findings are not sufficient in and of themselves to advise younger women with normal BMI to forego colonoscopic screening. Future studies that compare the efficacy of colonoscopy with other modalities in a prospective setting will address this issue in selected subject subgroups.

\section{References}

1. Winawer SJ, Zauber AG, Ho MN, et al. Prevention of colorectal cancer by colonoscopic polypectomy. The National Polyp Study Workgroup. N Engl J Med. 1993;329:1977-1981.

2. Zauber AG, Winawer SJ, O'Brien MJ, et al. Colonoscopic polypectomy and long-term prevention of colorectal-cancer deaths. $N$ Engl J Med. 2012;366:687-696.

3. Lieberman DA, Rex DK, Winawer SJ, Giardiello FM, Johnson DA, Levin TR. United States Multi-Society Task Force on Colorectal Cancer. Guidelines for colonoscopy surveillance after screening and polypectomy: a consensus update by the US MultiSociety Task Force on Colorectal Cancer. Gastroenterology. 2012;143:844-857.

4. Atkin WS, Valori R, Kuipers EJ, et al. International Agency for Research on Cancer. European guidelines for quality assurance in colorectal cancer screening and diagnosis. First Edition-Colonoscopic surveillance following adenoma removal. Endoscopy. 2012;44(Suppl 3):SE151-SE163.

5. Sonnenberg A, Delco F, Inadomi JM. Cost-effectiveness of colonoscopy in screening for colorectal cancer. Ann Intern Med. 2000;133:573-584.

6. Young PE, Womeldorph CM. Colonoscopy for colon cancer screening. J Cancer. 2013;4:217-226.

7. de Jonge V, Sint Nicolaas J, van Baalen O, et al. SCoPE consortium. The incidence of 30-day adverse events after colonoscopy among outpatients in the Netherlands. Am J Gastroenterol. 2012;107:878-884.

8. Brenner H, Haug U, Arndt V, Stegmaier C, Altenhofen L, Hoffmeister M. Low risk of colorectal cancer and advanced adenomas more than 10 years after negative colonoscopy. Gastroenterology. 2010;138:870-876.

9. Czwornog J, Austin GL. Body mass index, age, and gender affect prep quality, sedation use, and procedure time during screening colonoscopy Dig Dis Sci. (Epub ahead of print). doi:10.1007/ s10620-013-2746-2.

10. Schoenfeld P, Cash B, Flood A, et al. CONCeRN study investigators. Colonoscopic screening of average-risk women for colorectal neoplasia. N Engl J Med. 2005;352:2061-2068. 